\title{
Classical model of negative thermal expansion in solids with expanding bonds
}

\author{
Joseph T. Schick* \\ Department of Physics, Villanova University, Villanova, PA 19085, USA \\ Andrew M. Rappe \\ The Makineni Theoretical Laboratories, Department of Chemistry, \\ University of Pennsylvania, Philadelphia, Pennsylvania 19104-6323, USA
}

(Dated: September 17, 2018)

\begin{abstract}
We study negative thermal expansion (NTE) in model lattices with multiple atoms per cell and first- and second-nearest neighbor interactions using the (anharmonic) Morse potential. By exploring the phase space of neighbor distances and thermal expansion rates of the bonds, we determine the conditions under which NTE emerges. By permitting all bond lengths to expand at different rates, we find that NTE is possible without appealing to fully rigid units. Nearly constant, large-amplitude, isotropic NTE is observed up to the melting temperature in a classical molecular dynamics model of a $\mathrm{ReO}_{3}$-like structure when the rigidity of octahedral units is almost completely eliminated. Only weak NTE, changing over to positive expansion is observed when the corner-linked octahedra are rigid, with flexible second-neighbor bonds between neighboring octahedra permitting easy rotation. We observe similar changes to thermal expansion behavior for the diamond lattice: NTE when secondneighbor interactions are weak to positive thermal expansion when second-neighbor interactions are strong. From these observations, we suggest that the only essential local conditions for NTE are atoms with low coordination numbers along with very low energies for changing bond angles relative to bond-stretching energies.

PACS numbers: 65.40.-b,65.40.De
\end{abstract}

\section{INTRODUCTION}

Negative thermal expansion (NTE) is of great technological interest because of the potential to control or eliminate thermal stresses in real objects and devices. The material $\mathrm{ZrW}_{2} \mathrm{O}_{8}$ has been at the focus of intensive study because it exhibits substantial isotropic NTE over a wide temperature range ${ }^{1-4}$ While there is an expanding literature on the microscopic causes of NTE in this material, no model has proven completely adequate in making this connection. ${ }^{3,5-16}$

The picture at the heart of the discussion in $\mathrm{ZrW}_{2} \mathrm{O}_{8}$ is that the essential ionic motions leading to thermal changes in the lattice dimensions can be described by collective rotations of groups of atoms, the foundation of the "rigid unit model" (RUM), ${ }^{13}$ the rigid units being composed of $\mathrm{WO}_{4}$ tetrahedra and $\mathrm{ZrO}_{6}$ octahedra. Citing the exceptional rigidity of the $\mathrm{Zr}-\mathrm{O}-\mathrm{W}$ lines in the lattice seen in x-ray absorption fine-structure experiments, yet indicating distortion of the polyhedra, Cao and coauthors created a variation of the RUM labeled the "tent model," in which lattice contraction is a result of coherent motion of larger groups of atoms in the lattice. ${ }^{10,11}$ This disagreement in interpretation motivated a molecular dynamics study, ${ }^{16}$ employing empirical potentials from previous work, ${ }^{5}$ leading to the conclusion that the only essentially rigid units in the lattice are individual $\mathrm{W}-\mathrm{O}$ and $\mathrm{Zr}-\mathrm{O}$ bonds, questioning the validity of both the RUM and tent models. ${ }^{16}$

NTE has been observed via x-ray powder diffraction ${ }^{17}$ and coherent neutron powder diffraction experiments in $\mathrm{Li}_{2} \mathrm{~B}_{4} \mathrm{O}_{7} \cdot{ }^{18,19}$ The expansion in this material is anisotropic, with the contraction of one lattice parameter dominating the low-temperature volume dependence. The Li ions show strong $T$ dependence of their meansquared displacements around $100 \mathrm{~K}$, temperature at which NTE vanishes in the material. In the NTE regime, it is observed that the angle formed by $\mathrm{Li}-\mathrm{Li}-\mathrm{Li}$ chains correlates with the contracting lattice parameter, ${ }^{17,18}$ indicating the contribution of bond-bending to NTE.

Materials with simpler structure also exhibit NTE. For example, ranges of NTE are experimentally observed in diamond/zincblende structured materials Si, Ge, GaAs, GaSb, InAs, and InSb. ${ }^{20}$ In a comparative numerical study of models for NTE in $\mathrm{CuO}_{2}$, classical potentials were used to build three vibrational models based on rigid polyhedra, or rigid two- or three-atom rods. ${ }^{21}$ The resulting phonon dispersion curves were compared to the $a b$ initio curves, with results suggesting that the lowenergy modes correspond to rigid $\mathrm{Cu}-\mathrm{O}$ rods. ${ }^{21}$ In a density functional theoretical study of NTE in $\mathrm{Ag}_{2} \mathrm{O}, \mathrm{Cu}_{2} \mathrm{O}$, and $\mathrm{Au}_{2} \mathrm{O}$, the variation in NTE across these compounds is observed to correlate with the degree to which charge density is spherically distributed around the atoms, again supporting the importance of bond bending as a contributing factor in NTE.

$\mathrm{ReO}_{3}$ consists of a cubic lattice of octahedral units centered on Re ions. NTE is observed in the material over two temperature ranges, from $2 \mathrm{~K}$ to $220 \mathrm{~K}$ and from $600 \mathrm{~K}$ to $680 \mathrm{~K}$, in an experiment that includes temperatures up to about $800 \mathrm{~K}^{22}$ Experimental evidence supports $\mathrm{ReO}_{3}$ maintaining its cubic phase $(P m \overline{3} m)$ down to lowest temperatures ${ }^{23}$ and up to $800 \mathrm{~K}$ temperature. ${ }^{22}$ It has been suggested that NTE in $\mathrm{ReO}_{3}$ is readily explained within the RUM. ${ }^{22}$ These neutron 
studies indicate that O-atom motion transverse to Re-O bonds is significant and increases with temperature while longitudinal motion of the $\mathrm{O}$ atoms is minimal and independent of temperature, as evidenced by analysis of the mean-squared displacements of the ions. ${ }^{22}$ While this evidence supports treating $\mathrm{ReO}_{3}$ octahedral units as rigid, attributing the contraction of the lattice to coherent rotations of the octahedra, it does not eliminate the possibility of octahedral distortions that preserve the Re-O distances. Lending support to this view are the results of experimental and theoretical work reported on isostructural $\mathrm{ScF}_{3}$, in which the strong NTE observed is explained partly in terms of the distortions of the $\mathrm{ScF}_{6}$ octahedra. ${ }^{24}$ Additionally, the expansion of the Sc-F bonds with temperature is demonstrated in a recent density-functional molecular dynamics study that produces thermal expansion of $\mathrm{ScF}_{3}$, comparing well with experimental results. ${ }^{25}$

All vibration in materials is based on the fact that atoms sit in potential wells determined by their interactions with their neighbors, i.e. through the intervening chemical bonds. As the temperature is increased, the nuclei vibrate with greater amplitude. Because of the strong repulsion between atoms at close range, the potential between atoms is anharmonic. Thermal vibrations of atoms interacting through such an anharmonic potential generally drive neighboring atoms farther apart as temperature increases. In solids, the expansion of bonds is evident as thermal expansion. So how can contraction arise?

Especially as noted above in the discussion of modeling NTE in $\mathrm{ZrW}_{2} \mathrm{O}_{8}$, no simple model proves to be universally capable of explaining all NTE behavior, leading us to consider that by resolving a material into these units, some essential physics may have been neglected. Perhaps another approach may shed light on the situation. One aspect common to any rigid unit related model is the assumption that expansion of some bonds can be neglected, while molecular dynamics calculations indicate that more flexibility is necessary. ${ }^{16}$ A model without the rigidity requirement may help connect the influence of all-bond expansion to NTE.

Theoretical investigations of the general features of NTE include an analytical calculation of rigid corner linked squares ${ }^{26}$ and of rigid squares and octahedra joined together with flexible links, ${ }^{27}$ demonstrating that such models can exhibit NTE as well as elastic constants that decrease with temperature and pressure. In an alternative model, Rechtsman et al. devise an isotropic interaction potential that leads to NTE at low temperatures in close-packed structures of single-component many-particle systems, an effect demonstrated through constant-pressure Monte Carlo simulations. ${ }^{28}$ Their designed potential rises rapidly as the components (or atoms) move farther apart and less rapidly as they move closer together, in contrast to the commonly-accepted negative anharmonicity.

In this paper we present an investigation of model materials consisting of atoms all linked by bonds of vari-

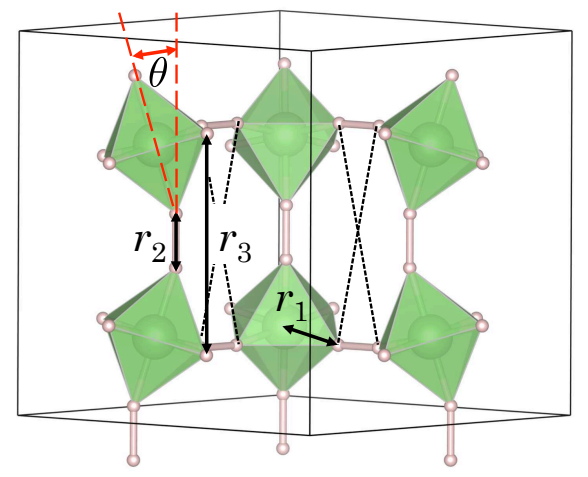

FIG. 1. (Color online) One unit cell of the three-dimensional model structure contains eight octahedra, connected by adjustable $r_{2}$ links, and pairs of second-neighbor links to provide the means to rotate the octahedra. A staggered arrangement of the second-neighbor links enables rotation of the rigid octahedra. The lengths of bonds between the center and vertex of an octahedron is $r_{1}, r_{2}$ is the distance of the link between octahedra, and $r_{3}$ is the distance between the pairs of parallel links being pushed apart by the second-neighbor interactions indicated with dashed lines. Only two pairs of second-neighbor links are shown in order to enhance clarity.

able flexibility. The flexibility is implemented through the Morse potential, ${ }^{29}$ providing known thermal expansion between atoms. (We note that NTE has been documented in an early lattice dynamics study employing several pair potentials, including the Morse potential. ${ }^{30}$ ) We show how the ratios of interatomic equilibrium lengths and their temperature dependences control the presence and extent of NTE. We examine three-dimensional models that are designed to illuminate the physics; we explore the relationships to existing simpler analytic models, the effect of enhanced flexibility of all bonds, and relationships to real NTE materials.

\section{MODELS}

We employ a three-dimensional model (Fig. 1) that bears some resemblance to previous two- and threedimensional work, ${ }^{11,26,27}$ however we replace fully rigid units with seven-atom octahedra. The octahedra form a cubic lattice, with interactions (bonds) between pairs of atoms represented by Morse potentials, ${ }^{29}$ which exhibit thermal expansion. Each now-flexible octahedron possesses interactions between the central atom $(A)$ and each of the six vertex atoms $(X)$. The rigidity of the octahedra is controlled by interactions between each $X$ atom and the four nearest $X$ atom vertices on the same octahedron. Second-neighbor interactions between $X$ atoms of different octahedra provide bond-bending stiffness, stabilizing the lattice. The center-to-vertex distance of an octahedron is denoted $r_{1}$. The distances between the vertices of nearest-neighbor octahedra are denoted as $r_{2}$, and 
the second- neighbor bonds as dashed lines. We specify the second-neighbor distance in terms of its projection $r_{3}$ onto the nearest Cartesian axis. When we perform calculations for corner-connected octahedra, $r_{2}=0$, the projection $r_{3}$ coincides with the second-neighbor interactions, and atoms are removed from the calculation to avoid doubled atoms at the vertices.

\section{A. Rigid mechanical model}

We analytically examine the effects of permitting expansion of the second-neighbor bonds only, treating them as expanding rods that rotate rigidly-linked and rigid octahedra. Rotation would not be permitted with these rigid rods if they coincide with the bonds defined above because the symmetrically arranged secondneighbor links between octahedra would compete with each other. To permit coherent rotation of the octahedra, we temporarily eliminate all but selected secondneighbor interactions. One set of these second-neighbor interactions lies in the $x z$-plane, with the long direction along the $z$-axis (see Fig. 1). Two additional similar sets of second-neighbor interactions lie in the $y z$-plane with a long $z$-axis and in the $x y$-plane with a long $x$-axis. Because of the asymmetry of this arrangement, the rotation pattern represents a tetragonal distortion of the unit cell (space group $I 4 / \mathrm{mmm}$ ). The contraction of the lattice parameters are functions of the angle $\theta$ describing the coherent rotations of octahedra about axes along alternating $\langle 110\rangle$ directions. To simplify the expressions below, we define reduced parameters $\tilde{r}_{2}=r_{2} / r_{1}$ and $\tilde{r}_{3}=r_{3} / r_{1}$. The relation between $\theta$ and bond lengths, normalized to $r_{1}$, is

$$
\tilde{r}_{3}=\tilde{r}_{2}+\sqrt{6} \cos \left(\theta-\theta_{\max }\right),
$$

where $\theta_{\max }=\tan ^{-1}(\sqrt{2} / 2) \approx 35.26^{\circ}$ is the angle for which $r_{2}$ bonds lie in planes formed by parallel secondneighbor bonds. In this configuration, there will be no torque produced by further expansion of second-neighbor bonds. If the second-neighbor bonds are to continue expanding, the octahedra and/or $r_{2}$ bonds would be required to distort or expand. (The limits on $\tilde{r}_{3}$ are $2+\tilde{r}_{2} \leq \tilde{r}_{3} \leq \sqrt{6}+\tilde{r}_{2}$. Reducing $\tilde{r}_{3}$ to less than $2+\tilde{r}_{2}$ simply reverses the sense of rotation of the octahedra, also yielding a contraction of the lattice.) The unit cell parameters in terms of the rotation, also normalized to $r_{1}$, are

$$
\tilde{a}=\tilde{b}=2\left(\cos \theta+\tilde{r}_{2}+1\right)
$$

and

$$
\tilde{c}=2\left(2 \cos \theta+\tilde{r}_{2}\right) .
$$

The dimensionless volume of the unit cell within the physically allowed limits is

$$
\widetilde{V}=8\left(\cos \theta+\tilde{r}_{2}+1\right)^{2}\left(2 \cos \theta+\tilde{r}_{2}\right) .
$$

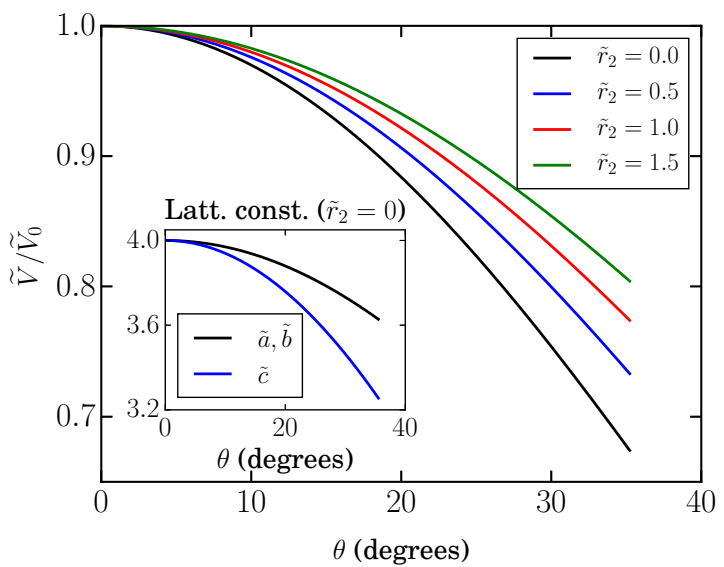

FIG. 2. (Color online) The relative volume of the threedimensional model with tetragonal distortions due to octahedral rotations. The maximum rotation angle $\left(\approx 35.3^{\circ}\right)$ corresponds to alignment of parallel $\tilde{r}_{2}$ and $\tilde{r}_{3}$ links, rendering further octahedral rotation impossible. The magnitude of volume contraction is maximal when the octahedra are corner-connected. The contraction for small angle rotation is vanishingly small. See Fig. 1 for a depiction of the angle. Inset: Normalized lattice constants of the corner-connectedeight-octahedra unit cell showing its tetragonal distortion for non-zero octahedral rotations are visible.

In Fig. 2 we plot $\widetilde{V} / \widetilde{V}_{0}$ as a function the rotation $\theta$ for several values of $\tilde{r}_{2}$. Because the octahedra are rotating into the available open space of the lattice, the greatest possible fractional contraction corresponds to the condition of corner-sharing octahedra, $\tilde{r}_{2}=0$. However, even if $\tilde{r}_{2} \approx 1$, substantial contraction is accessible. Because the slopes of the curves in Fig. 2 are vanishingly small for small octahedral rotations, lattice contraction is only possible if the rotations are sufficiently large enough to compensate for any expansion of the octahedra.

The present model in the limit of corner-shared octahedra $\left(\tilde{r}_{2}=0\right)$ is identical to the structure of $\mathrm{ReO}_{3}$. The discussion above suggests that the RUM model for NTE in $\mathrm{ReO}_{3}$ requires the rotations of the octahedra to be large enough to compensate for any expansion of the octahedral length. (Although in the present simple rigid mechanical model cubic symmetry is broken, it must be true that any rigid unit rotation causes volume contraction proportional to the cosine of some angle of rotation, which will have a similar vanishingly small effect for small rotations.) The changeover to positive thermal expansion (PTE) is understandable as being a result of octahedral expansion overwhelming the contracting effect of rotations. If octahedral expansions have begun to overwhelm the effect of rotations at $220 \mathrm{~K}$, then what might cause the observed return to NTE in the $600 \mathrm{~K}$ to $680 \mathrm{~K}$ range? More generally, we ask if there are microscopic material properties that might be manipulated to control the magnitude and temperature range of NTE? While the rigid mechanical model presented thus far has provided some 
TABLE I. Morse potential parameters used in molecular dynamics simulations presented in this work.

\begin{tabular}{|c|c|c|c|}
\hline Bond & $D(\mathrm{eV})$ & $\alpha\left(\AA^{-1}\right)$ & $r_{0}(\AA)$ \\
\hline \multicolumn{4}{|c|}{ Non-corner-connected rigid octahedra } \\
\hline$A-X$ & 5.00 & 5.53 & 2.00 \\
\hline$X-X\left(1^{\text {st }}\right.$ neighbor-onsite $\left.{ }^{\mathrm{a}}\right)$ & 5.00 & 5.53 & 2.83 \\
\hline$X-X\left(1^{\text {st }}\right.$ neighbor-offsite $\left.{ }^{\mathrm{b}}\right)$ & 5.00 & 5.53 & 1.50 \\
\hline$X-X\left(2^{\text {nd }}\right.$ neighbor $)$ & 5.00 & 5.00 & 5.70 \\
\hline \multicolumn{4}{|c|}{ Corner-connected rigid octahedra } \\
\hline$A-X$ & 5.00 & 5.53 & 2.00 \\
\hline$X-X\left(1^{\text {st }}\right.$ neighbor $)$ & 5.00 & 5.53 & 2.83 \\
\hline$X-X\left(2^{\text {nd }}\right.$ neighbor $)$ & 5.00 & 0.50 & 4.00 \\
\hline \multicolumn{4}{|c|}{ Corner-connected flexible octahedra } \\
\hline$A-X$ & 5.00 & 5.53 & 2.00 \\
\hline$X-X\left(1^{\text {st }}\right.$ neighbor $)$ & 0.30 & 0.50 & 2.83 \\
\hline$X-X\left(2^{\text {nd }}\right.$ neighbor $)$ & 0.30 & 0.50 & 4.00 \\
\hline
\end{tabular}

${ }^{\mathrm{a}} X$ atoms on the same octahedron. ${ }^{\mathrm{b}} X$ atoms on different octahedra.

insight, the dynamics of these simple systems is necessary to gain a more complete understanding.

\section{B. Classical molecular dynamics}

Even though octahedra are relatively stiff, they still expand and/or distort as a result of thermal motions. In order to assess the effects of these distortions on the volume of the crystal, we implemented molecular dynamics simulations for the present three-dimensional model using LAMMPS. ${ }^{31}$ Interactions between atoms were modeled with Morse potentials, with energy given by

$$
E_{\text {Morse }}(r)=D\left[e^{-2 \alpha\left(r-r_{0}\right)}-2 e^{-\alpha\left(r-r_{0}\right)}\right]
$$

where $D$ is the dissociation energy, $\alpha$ controls the width of the well, and $r_{0}$ is the zero temperature equilibrium distance. The parameters used in this work are displayed in Table I. We employ strong Morse bonds between the central $A$ atom of the octahedra and the $X$ atoms forming the octahedral vertices. To provide controllable rigidity of the octahedra, we also include Morse interactions between each $X$ atom and its four nearest-neighbor $X$ atoms on the same octahedron. We performed two separate sets of calculations with different arrangements of second-neighbor interactions. In the first case, we include only second-neighbor interactions between specific pairs of second-neighbor $X$ atoms corresponding to the mechanical links that we described in Section II A. In the second case, we include interactions between each $X$ atom and all six of its second nearest-neighbor $X$ atoms in a lattice of corner-connected octahedra.

Simulations, at fixed number, temperature, and pressure, were run for $13 \mathrm{~ns}$ (6.5 million steps) in supercells

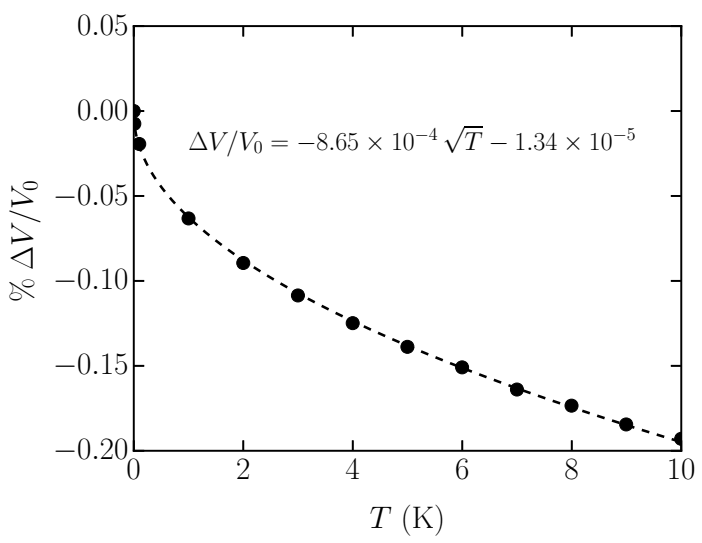

FIG. 3. The volume decreases from the reference volume as the square root of the temperature $T$. The dashed line represents the fit of the data to the square root of the temperature. Above the temperature displayed, thermal motions drive the system into a much lower volume as a result of an unstable arrangement of the pattern of second-neighbor bonds. We note that zero-point motion would be important in an accurate treatment at these temperatures.

consisting of 19,208 atoms and 65,856 bonds. In all cases, the starting configuration was at the the global minimum of the potential energy, corresponding to the maximum volume arrangement, with octahedral vertices aligned with the cubic supercell lattice directions. Average values of the lattice parameters were computed by sampling every 10,000 steps after the first two million steps.

\section{Tetragonal second-neighbor bond arrangement}

Simulations for octahedra without shared corners readily exhibited NTE. (See the examples in Figs. 3 and 4.) The volume change with $T$ is found to be proportional to $\sqrt{T}$. We studied arrangements of the second-neighbor interactions in our models. In one arrangement, there remained a near-zero-energy pathway for the octahedra to rotate, which led to a collapse of the lattice to a low volume configuration. At low enough temperatures, the collapse is avoided and NTE is observed; above the collapse temperature, NTE is still found. The collapse is a result of vibrationally-induced coherent shifts of layers of octahedra in an alternating pattern along a $\langle 100\rangle$ direction in the lattice. Above the collapse, the NTE coefficient is lower. In the second arrangement of second-neighbor bonds, the instability is removed and NTE is retained, with the supercell contracting tetragonally, similar to the rigid mechanical modeling discussed above. However, although the number of bonds and their parameters are the same for both the unstable and stable lattices, the magnitude of NTE of the stable lattice is smaller than that of the unstable lattice by a factor of $\sim 3.3$. Evidently, the instability assists NTE. 


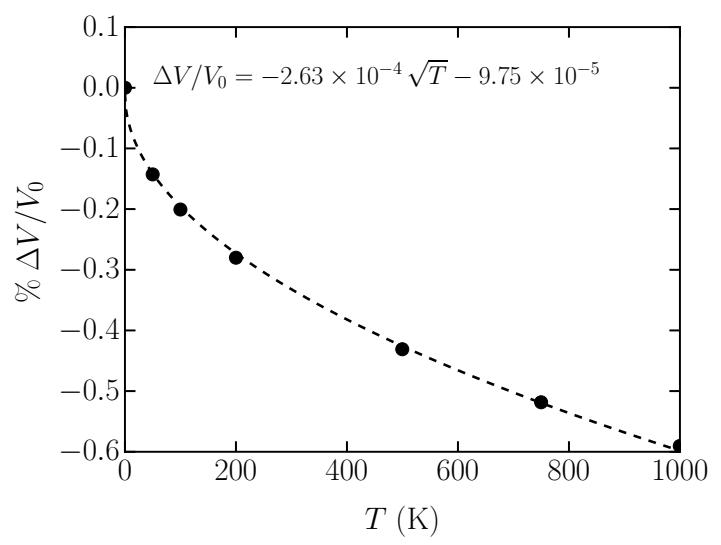

FIG. 4. Second-neighbor bond arrangement was updated to ensure lattice stability. Again, the volume decreases from the reference volume as the square root of the temperature $T$, however the magnitude of expansion is somewhat less than that displayed in Fig. 3. The dashed line is a fit of the data to the square root of the temperature. We note that the magnitude of NTE is nearly 3.3 times smaller than it is for the unstable lattice (see Fig. 3).

The positions of the octahedral vertex $X$-atoms are distributed more broadly perpendicular to the $A$ $X$ bonds, with transverse mean-squared displacements about five times larger than the mean-squared displacements along the bond. Furthermore, the transverse mean-squared displacements also display a temperature dependence proportional to $\sqrt{T}$. Angular deviations of the bonds grow with temperature, deviating by a mean of about $2^{\circ}$ and a standard deviation of about $1^{\circ}$ at the highest temperature shown in Fig. 4, consistent with larger motions of the vertex ions in the directions transverse to the bonds to central atoms.

A lattice of corner-sharing octahedra was also constructed, corresponding to the structure of $\mathrm{ReO}_{3}$. Also included were selected second-neighbor interactions imposing tetragonal symmetry. Simply transferring the Morse potential parameters that produced NTE in the case of separated octahedra resulted in positive thermal expansion (PTE), with the volume linearly increasing with temperature; the octahedral expansion clearly had overwhelmed any contraction due to rotations. We changed the Morse parameters of the second-neighbor interaction, reducing the restoring force to $\approx 1 \%$ of the first-neighbor restoring force. Subsequent simulations produced weak NTE that gives way to PTE as the temperature is increased (Fig. 5). This behavior is strikingly similar to the low-temperature thermal expansion in many materials that exhibit NTE, especially the case of $\mathrm{ReO}_{3} \cdot{ }^{22}$

The volume contraction behavior $(\propto \sqrt{T})$ indicates a relationship to the typical speeds of the particle motions or, equivalently, to the typical mean-squared displacements of the atoms bound in a Morse potential. We also note the above observation that the velocities

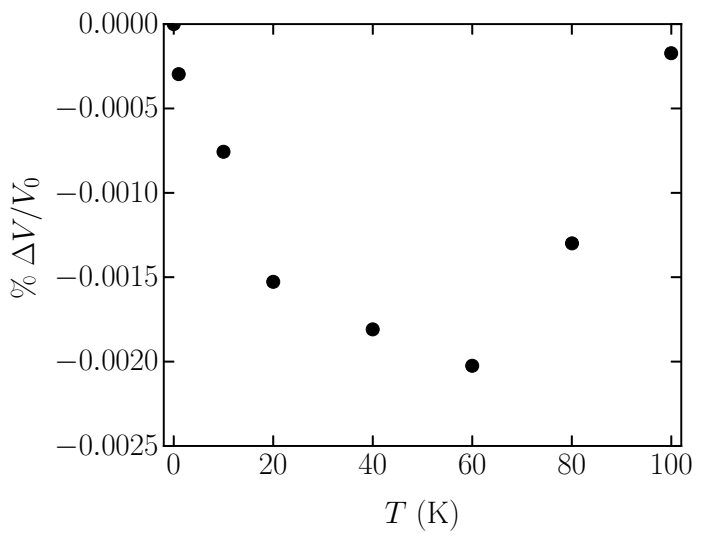

FIG. 5. Percentage relative volume change is plotted as a function of temperature $T$ using the Morse parameters for corner-connected rigid octahedra (see Table I). The lattice of corner-sharing rigid octahedra results in a thermal expansion curve moving from (weak) NTE to PTE, a distribution typical of many NTE materials. Further calculations at higher $T$ resulted in strong PTE.

of vertex atoms are biased into directions perpendicular to their bonds with the central atoms of the octahedra. The bond-expansion inherent in the Morse potential provides the force that drives atoms farther apart. ${ }^{29}$ In the present model, the nearest-neighbor coordinations, combined with weak second-neighbor interactions, guide the vertex atoms into the open spaces of the lattice, resulting in the observed NTE. In shared-corner octahedral structures, the transverse motions become overwhelmed by the expansion of bonds to the central atoms at sufficiently high temperatures, leading to the eventual change-over to positive thermal expansion. Adding links that separate the octahedra enables stronger NTE and pushes to much higher $T$ the eventual changeover to positive thermal expansion.

\section{Isotropic second-neighbor interactions}

When all interactions between $X$-atoms and their six second-neighbor vertex $X$-atoms for corner-connected octahedra are included, eliminating the artificially imposed tetragonal symmetry, NTE is still observed. In this arrangement, lattice contraction is only possible when the strength of the $X-X$ nearest-neighbor interaction is reduced to the same magnitude as the second-neighbor interactions, producing readily deformable octahedra and compromising the stability of the lattice. The NTE observed is isotropic, has a strong magnitude, and exists over wide temperature range up to the point where the material begins to melt (see Fig. 6). We observe that NTE is linear in temperature, contrasting with the behavior for the results displayed above. The mean-squared displacements of the vertex atoms transverse to their nearest-neighbor bonds expand linearly in temperature, 


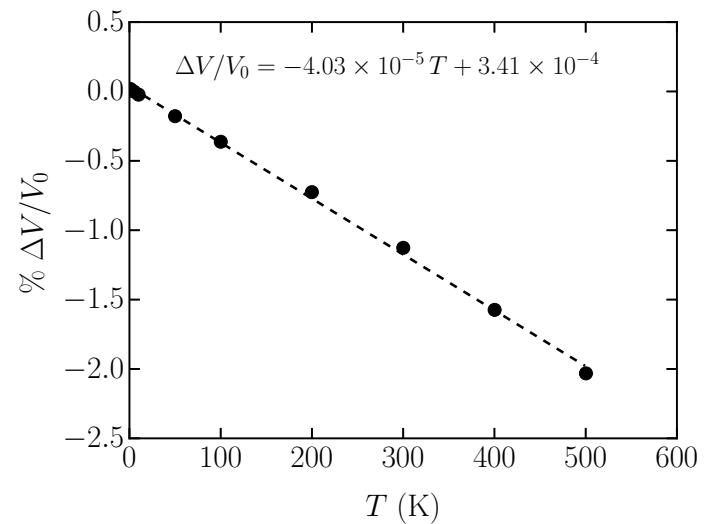

FIG. 6. Percentage relative volume change is plotted as a function of temperature corner-connected flexible octahedra. All vertex atoms interact with all six second-neighbor vertex atoms. Weak first- and second-neighbor interactions produce substantial NTE over a wide temperature range. A straight line is fit to the data.

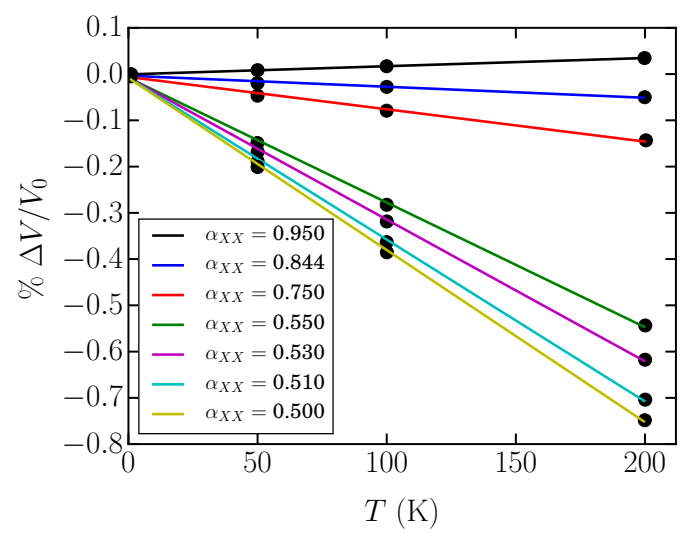

FIG. 7. (Color online) Percentage relative volume change is plotted as a function of temperature for several $X-X$ interaction strengths, as controlled by the Morse $\alpha$ parameter. Thermal expansion is shown to vary from NTE to PTE as the strength of the $X-X$ interactions increase. In all these plots, the well depths are held fixed at the values used for corner-connected flexible octahedra.

contrasting with the square-root dependence observed for rigid octahedra. The linearity of NTE in temperature is a result of the octahedra being much more flexible to angular distortions than in the examples above. Linearity is also a characteristic of the thermal expansion of a twoatom system bonded in the Morse potential. Varying the strength of the $X-X$ interactions, by adjusting the Morse $\alpha$ (see Eq. 5) permits control of thermal expansion in the model from NTE to PTE (see Fig. 7).

\section{DISCUSSION}

The common feature across all the materials exhibiting NTE is an open lattice. However, the fact that different materials with the same lattice possess different thermal expansion characteristics demonstrates the importance of bond strength ratios to thermal expansion. Bonding is essential in determining the equilibrium structure of a material. In addition, when there are competing phases with low enough energy differences, states with higher entropy will be favored as temperature increases. The present model calculations, in which the structural stability is maintained by second-neighbor interactions, demonstrate that reducing the strength of secondneighbor interactions leads to NTE, which possibly is due to increased accessibility to competing phases. Materials with large unit cells, such as $\mathrm{ZrW}_{2} \mathrm{O}_{8}$ and $\mathrm{Li}_{2} \mathrm{~B}_{4} \mathrm{O}_{7}$ readily provide support for this picture. $\mathrm{ZrW}_{2} \mathrm{O}_{8}$ is known to be metastable ${ }^{32}$ and metastability such as this has been cited as a likely underlying reason for NTE. ${ }^{33}$ It is furthermore noted that synthesis of good $\mathrm{Li}_{2} \mathrm{~B}_{4} \mathrm{O}_{7}$ samples is hampered by the existence of a number of (meta)stable phases under growth conditions. ${ }^{18}$

In contrast to these complex materials, which have competing metastable states and provide one view of NTE, we point out that NTE is observed even in the simplest materials, such as those with diamond or other low-coordination lattices. In early lattice dynamics modeling with spherically symmetric potentials, the diamond lattice was observed to exhibit NTE. ${ }^{30}$ We reproduced this observation in our own molecular dynamics calculations. Furthermore, we found that by increasing the strength of second-neighbor interactions, stabilizing the lattice, we can eliminate NTE in this lattice as we did for corner-connected octahedral model discussed above. Experimentally, NTE in $\mathrm{Ag}_{2} \mathrm{O}$ has a greater magnitude than in $\mathrm{Cu}_{2} \mathrm{O}$, while it is nearly nonexistent in $\mathrm{Au}_{2} \mathrm{O}$, and this magnitude was observed to correlate, in DFT calculations, with the degree to which the charge density is spherically distributed ${ }^{34}$ which again supports the view that lower directionality of bonds is important. In all of these examples as in our model calculation, the open lattice and the weak second-neighbor bond potentials, implying highly flexible bond angles, are the essential ingredients that yield NTE. The reasonable success of the RUM in describing NTE is a result of the fact that there are strong bonds within the polyhedra, minimizing their expansion, coupled with low resistance to rotations. We observe from the current calculations that reducing the resistance to changing bond angles within polyhedra yields stronger NTE behavior with a constant coefficient of expansion, such as that seen in $\mathrm{ZrW}_{2} \mathrm{O}_{8}$, lending further support to previous findings ${ }^{16}$ that models involving coherent motions of atoms, such as the RUM, are not consistent with the NTE in this material. Finally, from the observation in the current model that angular deviations within the octahedra is a key to the emergence of NTE leads us to suggest that the observed reappear- 
ance of NTE in $\mathrm{ReO}_{3}$ at higher temperature could be a result of temperature-induced reduced rigidity of the octahedra permitting more atomic motions to expand into remaining lower-density regions of the lattice. However, confirmation of this is beyond the scope of the present work.

\section{CONCLUSION}

We constructed model systems consisting of point masses joined by anharmonic springs, analytically showed that the RUM is a decent description of NTE as long as coherent rotations of the rigid units is sufficient to outweigh any uniform expansion of the same rigid units. We showed that NTE can be strongly enhanced by increasing the flexibility of previously considered to be rigid units. Finally, we demonstrated that a merely modest reduction in this flexibility is enough to restore positive expansion.

\section{ACKNOWLEDGMENTS}

The authors acknowledge the support of the Department of Energy, Office of Basic Energy Sciences, through grant DE-FG02-07ER46431. Computational support was provided by the National Energy Research Scientific Computing (NERSC) Center.
* joseph.schick@villanova.edu

1 C. Martinek and F. A. Hummel, J. Am. Ceram. Soc. 51, 227 (1968).

2 J. S. O. Evans, T. A. Mary, T. Vogt, M. A. Subramanian, and A. W. Sleight, Chemistry of Materials 8, 2809 (1996), http://dx.doi.org/10.1021/cm9602959.

3 T. A. Mary, J. S. O. Evans, T. Vogt, and A. W. Sleight, Science 272, 90 (1996), http://www.sciencemag.org/content/272/5258/90.full.pdf.

${ }^{4}$ F. R. Drymiotis, H. Ledbetter, J. B. Betts, T. Kimura, J. C. Lashley, A. Migliori, A. P. Ramirez, G. R. Kowach, and J. Van Duijn, Phys. Rev. Lett. 93, 025502 (2004).

5 A. K. A. Pryde, K. D. Hammonds, M. T. Dove, V. Heine, J. D. Gale, and M. C. Warren, J. Phys.: Condens. Matter 8, 10973 (1996).

6 A. P. Ramirez and G. R. Kowach, Phys. Rev. Lett. 80, 4903 (1998).

7 G. Ernst, C. Broholm, G. R. Kowach, and A. P. Ramirez, Nature 396, 147 (1998).

8 R. Mittal and S. L. Chaplot, Phys. Rev. B 60, 7234 (1999).

9 R. Mittal, S. L. Chaplot, H. Schober, and T. A. Mary, Phys. Rev. Lett. 86, 4692 (2001).

10 D. Cao, F. Bridges, G. R. Kowach, and A. P. Ramirez, Phys. Rev. Lett. 89, 215902 (2002).

11 D. Cao, F. Bridges, G. R. Kowach, and A. P. Ramirez, Phys. Rev. B 68, 014303 (2003).

12 J. N. Hancock, C. Turpen, Z. Schlesinger, G. R. Kowach, and A. P. Ramirez, Phys. Rev. Lett. 93, 225501 (2004).

13 M. G. Tucker, A. L. Goodwin, M. T. Dove, D. A. Keen, S. A. Wells, and J. S. O. Evans, Phys. Rev. Lett. 95, 255501 (2005).

14 V. Gava, A. L. Martinotto, and C. A. Perottoni, Phys. Rev. Lett. 109, 195503 (2012).

15 F. Bridges, T. Keiber, P. Juhas, S. J. L. Billinge, L. Sutton, J. Wilde, and G. R. Kowach, Phys. Rev. Lett. 112, 045505 (2014).

16 A. Sanson, Chemistry of Materials 26, 3716 (2014), http://dx.doi.org/10.1021/cm501107w.

17 N. Sennova, R. Bubnova, J. Shepelev, S. Filatov, and O. Yakovleva, Journal of Alloys and Compounds 428, 290
(2007).

18 A. Senyshyn, B. Schwarz, T. Lorenz, V. T. Adamiv, Y. V. Burak, J. Banys, R. Grigalaitis, L. Vasylechko, H. Ehrenberg, and H. Fuess, Journal of Applied Physics 108, 093524 (2010).

19 A. Senyshyn, H. Boysen, R. Niewa, J. Banys, M. Kinka, Y. Burak, V. Adamiv, F. Izumi, I. Chumak, and H. Fuess, J. Phys. D: Appl. Phys. 45, 175305 (2012).

20 P. W. Sparks and C. A. Swenson, Phys. Rev. 163, 779 (1967).

21 L. H. N. Rimmer, M. T. Dove, B. Winkler, D. J. Wilson, K. Refson, and A. L. Goodwin, Phys. Rev. B 89, 214115 (2014).

22 T. Chatterji, T. C. Hansen, M. Brunelli, and P. F. Henry, Appl. Phys. Lett. 94, 241902 (2009).

23 E. S. Božin, T. Chatterji, and S. J. L. Billinge, Phys. Rev. B 86, 094110 (2012).

24 C. W. Li, X. Tang, J. A. Muñoz, J. B. Keith, S. J. Tracy, D. L. Abernathy, and B. Fultz, Phys. Rev. Lett. 107, 195504 (2011).

25 P. Lazar, T. Bučko, and J. Hafner, Phys. Rev. B 92, 224302 (2015).

26 M. E. Simon and C. M. Varma, Phys. Rev. Lett. 86, 1781 (2001).

27 Y. He, V. Cvetkovic, and C. M. Varma, Phys. Rev. B 82, 014111 (2010).

28 M. C. Rechtsman, F. H. Stillinger, and S. Torquato, The Journal of Physical Chemistry A 111, 12816 (2007), pMID: 17988108, http://dx.doi.org/10.1021/jp0768591.

29 Supplemental Material.

30 D. C. Wallace and J. L. Patrick, Phys. Rev. 137, A152 (1965).

31 S. Plimpton, Journal of Computational Physics 117, 1 (1995).

32 A. K. Arora, V. S. Sastry, P. C. Sahu, and T. A. Mary, J. Phys.: Condens. Matter 16, 1025 (2004).

33 Z.-K. Liu, Y. Wang, and S.-L. Shang, Scripta Materialia 65, 664 (2011).

34 M. K. Gupta, R. Mittal, S. L. Chaplot, and S. Rols, Journal of Applied Physics 115, 093507 (2014). 


\title{
Classical model of negative thermal expansion in solids due to positively expanding bonds: Supplementary material
}

\author{
Joseph T. Schick \\ Department of Physics, Villanova University, Villanova, PA 19085, USA \\ Andrew M. Rappe \\ The Makineni Theoretical Laboratories, Department of Chemistry, \\ University of Pennsylvania, Philadelphia, Pennsylvania 19104-6323, USA
}

(Dated: September 17, 2018)

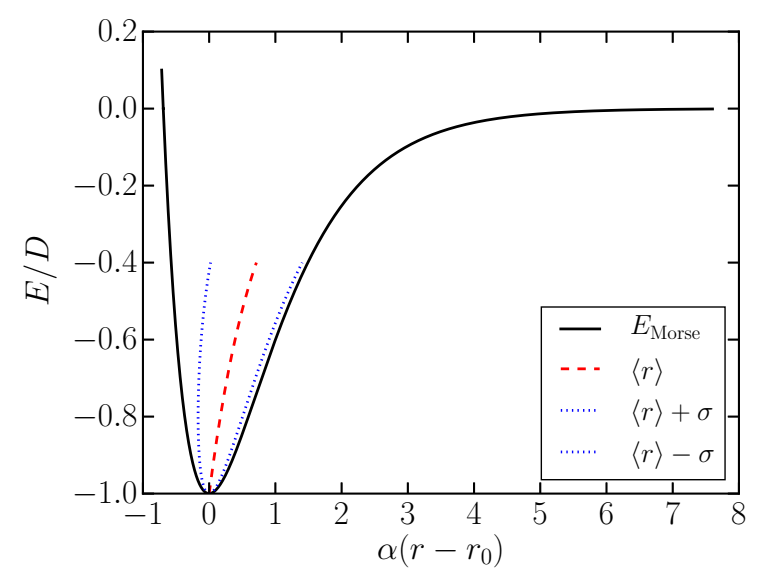

FIG. 1. The expansion of the one dimensional Morse oscillator is displayed in terms of energy normalized to $D$, which is the dissociation energy. The mean value of the displacement (red dashes) is evaluated analytically (Eq. 4) and the rootmean-squared deviations of the displacement (blue dots) are computed numerically from the equation of motion. Turning points of classical motion (black curves) are displayed for reference.

The Morse potential ${ }^{1}$ captures the essential anharmonicity necessary for describing the connection between bond expansion and thermal expansion properties of materials. Furthermore, the Morse potential has an easily calculable thermal expansion which makes it especially suitable for this model calculation. The Morse potential between two atoms separated by $r$ is given by the expression

$$
E_{\text {Morse }}(r)=D\left[e^{-2 \alpha\left(r-r_{0}\right)}-2 e^{-\alpha\left(r-r_{0}\right)}\right]
$$

where $r_{0}$ is the equilibrium separation, $D$ is the disso- ciation energy and $\alpha$, with dimension of inverse length, controls the width of the potential well.

The solution of the equation of motion of the classical Morse oscillator for bounded motion has a simple analytic form, (e.g. see Ref. 2),

$$
r(t)-r_{0}=\frac{1}{\alpha} \ln \left[\frac{1-\sqrt{\epsilon} \cos \omega t}{1-\epsilon}\right] ; \quad(\epsilon<1),
$$

where $\epsilon=\left(E_{\text {Morse }}+D\right) / D$ is the energy of the oscillator as a fraction of the dissociation energy $D$ and the angular frequency $\omega$ is given in terms of the potential parameters and the reduced mass $\mu$ by

$$
\omega^{2}=\frac{2 \alpha^{2} D}{\mu}(1-\epsilon)
$$

Integrating Eq. 2 over one period, we determine the mean displacement

$$
\left\langle r-r_{0}\right\rangle=\frac{1}{\alpha} \ln \left[\frac{1+\sqrt{1-\epsilon}}{2(1-\epsilon)}\right] .
$$

We plot this value along with numerically determined root-mean-squared deviations in Fig. 1.

We are interested in the low energy limit, at which Eq. 4 is found to be linear in energy,

$$
\left\langle r-r_{0}\right\rangle \approx \frac{3 \epsilon}{4 \alpha} .
$$

To obtain the low energy behavior of the mean-squared displacement, $\left\langle\left(r-r_{0}\right)^{2}\right\rangle$, we expand Eq. 2 to sixth order in $\sqrt{\epsilon}$ and then integrate to find

$$
\left\langle\left(r-r_{0}\right)^{2}\right\rangle=\frac{1}{\alpha^{2}}\left[\frac{1}{2} \epsilon+\frac{27}{32} \epsilon^{2}+O\left(\epsilon^{3}\right)\right],
$$

implying the dimensionless root-mean-squared displacement is $\sigma=\sqrt{\epsilon / 2}$ to lowest order in $\epsilon$.
1 P. M. Morse, Phys. Rev. 34, 57 (1929).
${ }^{2}$ W. C. DeMarcus, American Journal of Physics 46, 733 (1978). 